\title{
Expression of miR-224-5p is associated with the original cisplatin resistance of ovarian papillary serous carcinoma
}

\author{
HENAN ZHAO $^{1}$, TIE BI ${ }^{2}$, ZHENYUN QU $^{1}$, JIYONG JIANG ${ }^{2}$, SHIYING CUI $^{3}$ and YAN WANG ${ }^{4}$ \\ ${ }^{1}$ Department of Pathophysiology, Dalian Medical University; ${ }^{2}$ Obstetrics and Gynecology Hospital, Dalian; \\ ${ }^{3}$ Department of Human Histology and Embryology, Dalian Medical University; ${ }^{4}$ Laboratory Center, \\ Second Affiliated Hospital of Dalian Medical University, Dalian, P.R. China
}

Received March 26, 2014; Accepted June 5, 2014

DOI: 10.3892/or.2014.3311

\begin{abstract}
Chemoresistance is a major challenge to successful chemotherapy of ovarian cancer, which represents the leading cause of mortality from gynecologic malignancies. We demonstrated that overexpression of miR-224-5p in ovarian cancer patients is associated with platinum-based chemoresistance using miRNA microarray analysis and quantitative real-time polymerase chain reaction (qRT-PCR) validation in vivo, as well as in 4 human ovarian cancer cell lines (C13/OV2008; A2780CP/A2780S) in vitro. In the present study, we aimed to clarify the role of miR-224-5p in regulating the chemoresistance of ovarian cancer. By using the sensitive miRNA transient transfection, we demonstrated expression and bioactivity of miR-224-5p in ovarian cancer cell lines. It is of note that enforced expression of miR-224-5p enhanced chemoresistance to cisplatin in ovarian cancer cells through apoptosis reversion. We predicted and identified the PRKCD gene as one of the targets of miR-224-5p in mediating the primary chemoresistance of ovarian cancer patients. We showed reciprocal expression of miR-224-5p and PRKCD by quantitative analysis in complete response and incomplete response patients in vivo, and 2 pairs of cisplatin resistance and sensitive cell lines in vitro, after either miR-224-5p overexpression or knockdown transfection. Additionally, miR-224-5p and PRKCD can serve as novel predictors and prognostic biomarkers for ovarian papillary serous carcinoma (OPSC) patient response to overall disease-specific survival. Our findings suggest that miR-224-5p may function as an oncogene and induce platinum resistance in OPSC at least in part by downregulating PRKCD, thereby providing a biomarker for predicting chemosensitivity to cisplatin in patients with ovarian cancer.
\end{abstract}

Correspondence to: Professor Yan Wang, Laboratory Center, Second Affiliated Hospital of Dalian Medical University, 467 Zhongshan Road, Dalian 116023, P.R. China

E-mail: yanzi941026@126.com

Key words: ovarian cancer, serous carcinoma, platinum-resistant, cisplatin, microRNA, PRKCD

\section{Introduction}

Ovarian cancer is the most common cause of mortality from a gynecologic malignancy in the world, posing a serious risk to women's health. A previous report showed that, 28,082 women were diagnosed with epithelial ovarian cancer (EOC) from 1988 to 2001. The largest histology subgroup, 49.3\% $(13,835)$ of patients, had ovarian papillary serous carcinoma (OPSC) (1). Due to a lack of effective biomarkers as screening tests and the absence of symptoms at the early stage of this disease, more than two thirds of women with ovarian cancer have advanced disease (stage III or IV) at the time of diagnosis. Platinum (cisplatin or carboplatin) has been used as the first-line chemotherapeutic drug for ovarian cancer patients since its introduction in the mid-1970s (2), and platinum-based chemotherapy has been the standard mainstay treatment for advanced EOC (2); however, successful long-term treatment is prevented by the development of drug resistance (3). Moreover, empirically-based treatment strategies generally result in many chemotherapy-resistant patients receiving significant toxicities during multiple cycles of therapy. Therefore, it is clinically important to identify biomarkers that may assist in detecting and predicting which patients with ovarian cancer will respond to platinum-based chemotherapy and which patients will remain refractory to this standard treatment.

MicroRNAs (miRNAs) are short non-coding RNAs that act as post-transcriptional regulators; they can effectively silence target genes by binding to the 3'-untranslated region (3'-UTR) of target mRNAs, causing mRNA degradation or inhibiting translation (4). Increasing studies have indicated that miRNAs are not only involved in the development and progression of human cancers $(5,6)$, but they also play a vital role in tumor cell response to chemotherapeutic agents by acting as oncogenes and tumor suppressors (7). From the advances in the use of the miRNA microarray technique, which can provide gene phenotyping that identifies distinct classifications exceeding traditional histopathologic methods (8-11), another promising approach has emerged, the use of miRNAs encoded by the human genome as diagnostic tools to predict drug response.

In the present study, we applied this approach to identify miR-224-5p expression patterns strongly associated with the response to primary platinum-based chemotherapy. To understand the mechanism of miR-224-5p in regulating 
chemoresistance, we chose TargetScan, MicroCosm Targets and miRNA Target Visualization to find the possible target genes. After finding the common target genes from them, only the top significantly predicted targets were obtained, and PRKCD, whose gene product plays a role in both regulating chemoresistance and promoting apoptosis (4), became a possible target gene of miR-224-5p.

The purpose of the present study was to validate our microarray results and then couple this analysis with biofunctional variations that reflect and identify the regulations of various oncogenic-miRNA signaling pathways to identify a unique mechanism of platinum resistance that can guide the use of chemotherapy drugs in platinum-resistant EOC patients.

\section{Materials and methods}

Patients and samples. Patients who were surgically treated for ovarian cancer at the Obstetrics and Gynecology Hospital, Dalian, China, between July 2004 and November 2010, were identified. We evaluated a total of 41 OPSC samples that were resected at the time of primary surgery from patients who went on to receive platinum-based chemotherapy. The clinicopathological characteristics of the patients who contributed the ovarian cancer samples are listed in Table I. All pathological specimens were reviewed by two independent pathologists with no knowledge of patients' clinical data. The diagnosis of the cases was based on criteria of the International Federation of Gynecology and Obstetrics (FIGO) staging system.

Therapeutic response was evaluated as previously reported (12) from the medical record by a single gynecologic oncologist using standard criteria for patients with measurable disease, based on WHO guidelines $(12,13)$. A complete response (CR) was classified as a complete disappearance of all measurable and assessable disease or, in the absence of measurable lesions, a normalization of CA-125 levels following platinum-based therapy. An incomplete response (IR) described patients who exhibited only a partial response, had no response, or progressed during primary therapy (12). CA-125 response criteria were based on established guidelines and were used only in the cases with the absence of a measurable lesion $(12,14)$.

The experiments were performed with two different groups of human ovarian cancer cell lines, each group including one cisplatin-sensitive parental cell line (OV2008 and A2780S) and its cisplatin-resistant variant (C13 and A2780CP), respectively).

Cell culture, transfection and treatment. Ovarian cancer cells (A2780CP/A2780S and C13/OV2008) were cultured in RPMI-1640 medium (Invitrogen, Burlington, ON, Canada) supplemented with $10 \%$ fetal bovine serum (FBS) and maintained at $37^{\circ} \mathrm{C}, 5 \% \mathrm{CO}_{2}$. All tissue culture reagents were obtained from Sigma-Aldrich (St. Louis, MO, USA). The miR-224-5p mimics and inhibitors were designed and chemically synthesized by Ambion (cat. no. 4427975; Life Technologies Corporation, Denmark).

Lipofectamine 2000 (Invitrogen) was incubated with pre-miR-224-5p (miRNA mimic), anti-miR-224-5p (inhibitor) or their scrambled negative controls (Ambion; mock) at a concentration of $90 \mathrm{nmol} / 1$ and incubated in serum-free RPMI-1640 for 20 min before being added to OV2008/A2780S or $\mathrm{C} 13 / \mathrm{A} 2780 \mathrm{CP}$ cells, respectively. Cells were incubated at $37^{\circ} \mathrm{C}$ for $4 \mathrm{~h}$ before $10 \% \mathrm{FBS}$ was replaced. Protein and RNA were harvested $48 \mathrm{~h}$ after transfection. For cisplatin treatment, cells were maintained in medium with the desired doses of cisplatin (cat. no. P4394; Sigma, USA).

miRNA microarray and data analysis. A microarray platform optimized for the analysis of a panel of 768 human miRNAs was used to analyze and compare the patterns of miRNA expression between CR and IR ( $\mathrm{n}=4$ for all) to platinum-based chemotherapy in OPSC patients. Total RNA that was enriched for miRNAs was extracted from the FFPE tissue by using the Ambion mirVana microRNA Isolation kit (Ambion, USA). The quality of total RNA was assessed using the Agilent Bioanalyzer (Agilent Technologies, Santa Clara, CA, USA). Individual quantitative real-time polymerase chain reaction (qRT-PCR) assays were formatted into a TaqMan low-density array (TLDA; Applied Biosystems), which was performed at the Shannon McCormack Advanced Molecular Diagnostics Laboratory Research Services, the Dana Farber Cancer Institute, the Harvard Clinic and Translational Science Center. The normalized microarray data were managed and analyzed by StatMiner version 3.0 (Integromics ${ }^{\mathrm{TM}}$ ).

$R N A$ isolation and $q R T-P C R$. Total RNA was prepared using TRIzol reagent, following the manufacturer's instructions. qRT-PCR was performed using the TaqMan MicroRNA Reverse Transcription kit (Applied Biosystems, Foster City CA, USA) with ABI miRNA specific primers and primer kits on an Agilent Technologies Stratagene Mx3000P (USA). Specific kits used were: hsa-miR-224-5p; ABI no. 4427975. The products were detected with SYBR-Green I, and their relative miRNA or mRNA levels were calculated using the comparative cycle threshold $\left[\mathrm{Ct}, 2^{-\Delta \Delta \mathrm{Ct}}\right]$ method with U6 and GAPDH as the endogenous controls, respectively. Samples from at least three independent experiments, each measured in duplicate, were analyzed and the data are expressed as the averages $\pm \mathrm{SD}$.

MTS cell viability assay. The cytotoxic effect on cell survival was quantified using the CellTiter $96{ }^{\circledR} \mathrm{AQ}_{\text {ueous }}$ Non-Radioactive Cell Proliferation Assay kit (cat. no. P9625; Promega Co., USA). Briefly, cells were cultured in 96-well plates at a density of $1 \times 10^{3} /$ well for $48 \mathrm{~h}$ after transfection, and then treated with $0,5,10,15,20,30,40,50,60,70,85$ or $100 \mu \mathrm{mol} / 1$ cisplatin (cat. no. P4394; Sigma) for 96 h. MTS/PMS solution composed of $20 \mu \mathrm{l}$ of two reagents, a novel tetrazolium compound (MTS) and an electron coupling reagent (PMS), was added into one 96-well plate at a volume of $100 \mu \mathrm{l}$ with cultured cells, and further incubated at $37^{\circ} \mathrm{C}$ in a humidified, $5 \% \mathrm{CO}_{2}$ atmosphere for $1 \mathrm{~h}$. The absorbance at $490 \mathrm{~nm}$ was recorded using an ELISA plate reader (PowerWavex 340; Bio-Tek Instruments Inc., Winooski, VT, USA).

Cellular apoptosis assay by assessing the activation of caspase-3 and -7, and with TUNEL. Ovarian cancer cells were initially seeded at a concentration of $1 \times 10^{5}$ cells $/ \mathrm{ml}$, in 6 -well plates, and incubated at $37^{\circ} \mathrm{C}$ in a humidified atmosphere with $5 \% \mathrm{CO}_{2}$ for $48 \mathrm{~h}$ after transfection, then treated with $20 \mu \mathrm{mol} / 1$ cisplatin for C13 and OV2008, respectively. After 
exposure to the drugs for $48 \mathrm{~h}$, cell apoptosis was first determined by assessing the activation of caspase- 3 and -7 using the Caspase-Glo 3/7 Assay kit (Promega ${ }^{\mathrm{TM}}$ Co., Shanghai, China). After the cells were incubated in normal medium containing the Caspase-Glo reagent for $1 \mathrm{~h}$, caspase- 3 and -7 activities were detected with a luminometer. At the same time, the nuclear morphology of apoptotic cells was determined using the TUNEL In Situ Apoptosis Detection kit (KeyGen Biotech Inc., China), following the manufacturer's instructions. The apoptotic cells (brown staining) were counted under a microscope.

miRNA target prediction and pathway analysis. In order to understand the mechanism of miR-224-5p in regulating chemoresistance, several computational approaches were used to analyze target prediction of miRNAs, including miRDB (http:// mirdb.org/miRDB/), MicroCosm Targets (http://www.ebi. ac.uk/enright-srv/microcosm/htdocs/targets/v5/) and miRNA Target Visualization (https://cm.jefferson.edu/rna22v1.0homo_sapiens/GetInputs.jsp). Functional analysis of these predicted targets was performed to identify biologic pathways, according to significant gene expression. The target prediction algorithm used here is estimated to have a $20-30 \%$ false positive rate. This level of false discovery is unlikely to affect the overall network findings obviously, although the number of top predicted gene targets is large. Furthermore, functional analysis of these predicted gene targets may assist in identifying biologic pathways with significant involvement for gene expression.

Western blot analysis. Western blotting was performed as follows: harvested cells were lysed in lysis buffer (Beyotime Institute of Biotechnology, Shanghai, China) and the proteins $(20 \mu \mathrm{g})$ were separated on $10 \%$ SDS-PAGE gels and transferred to nitrocellulose membranes. Membranes were blocked in PBS containing $0.05 \%$ Tween-20 (TBST)-5\% non-fat milk. Then, the membrane was incubated with antibodies for PRKCD or GAPDH. After secondary antibody incubation, the signal analysis was performed by exposure of the blots to films, which were then scanned and band intensities were measured with Labworks-Analyst (GeneCo) software.

Statistical analysis. The results were analyzed by SPSS 17.0 (Chicago, IL, USA). The data are expressed as arithmetic mean \pm SD of the number (n) of experiments. Samples were analyzed with repeated measures analysis of variance, and differences in the incidences were analyzed using ANOVA. Statistical analysis of percent values was performed by the Pearson's $\chi^{2}$ test. The overall survival duration was defined as the interval (in months) between the date of initial cytoreductive surgery to date of last follow-up or death. The survival time courses were studied using the Kaplan-Meier method, and groups were compared using the log-rank test; $\mathrm{p}<0.05$ was considered to indicate a statistically significant difference.

\section{Results}

Patient characteristics. The clinicopathological characteristics of the patients who contributed the OPSC samples are listed in Table I. Forty-one patients were identified matching
Table I. Clinicopathological characteristics of ovarian cancer patients.

\begin{tabular}{lcc}
\hline Characteristics & $\begin{array}{c}\text { Clinical } \\
\text { complete } \\
\text { responders } \\
(\mathrm{n}=22)\end{array}$ & $\begin{array}{c}\text { Clinical } \\
\text { incomplete } \\
\text { responders } \\
(\mathrm{n}=19)\end{array}$ \\
\hline Mean age, years & 52.6 & 49.3 \\
Stage & & \\
I & 1 & 2 \\
II & 3 & 2 \\
III & 18 & 12 \\
IV & 0 & 3 \\
Grade & 2 & 1 \\
1 & 12 & 10 \\
2 & 8 & 8 \\
3 & & 1638.5 \\
Mean serum CA-125, $\mathrm{m} / \mathrm{ml}$ & 851.3 & 316.7 \\
Before platinum & 15.2 & $32.8^{\mathrm{a}}$ \\
After platinum & 50.8 & $57.9^{\mathrm{a}}$ \\
Mean survival time, months & 31.8 & \\
Mortality rate $(\%)$ & & \\
\hline
\end{tabular}

${ }^{\mathrm{a}} \mathrm{p}<0.05$, compared with complete response group.

the study criteria. Twenty-two OPSC patient samples demonstrated a CR, and 19 showed an IR to primary platinum-based therapy following the surgery. FFPE blocks were obtained after surgery.

miRNA microarray results and $q R T-P C R$ validation. To further characterize the unique miRNAs in OPSC differentiation, specimens from the CR and IR OPSC patients were initially analyzed by miRNA microarray, respectively. Of the 768 miRNAs analyzed by microarray, we identified miR-224-5p expression patterns were strongly downregulated in CR OPSC when compared with IR OPSC patients (Fig. 1A). In order to confirm microarray results, qRT-PCR validation was performed. RNA was isolated from a new set of FFPE tissues to increase the possibility that the observed differences in miRNA expression profiles represented biologically significant changes. In keeping with microarray results, miR-224-5p was low-expressed in CR OPSC with significance, and representative analysis is shown in Fig. 1B.

miR-224-5p expression in different chemoresistant ovarian cancer cell lines, and upregulation of miR-224-5p increases cell survival of chemosensitive cell lines to cisplatin. In the previous results, we demonstrated that miR-224-5p expression levels were significantly higher in IR tumor specimens than in CR tumors specimens (Fig. 1A and B). Thus, we sought to further verify the relationship between miR-224-5p expression and chemosensitivity using the human ovarian cancer cell lines OV2008 and A2780S, and their cisplatin-resistant variants, C13 and A2780CP, respectively. As indicated in Fig. 1C, 


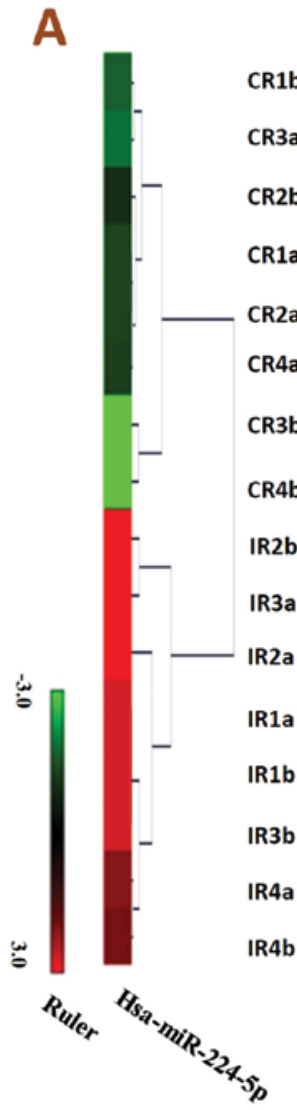

B

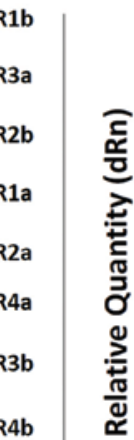
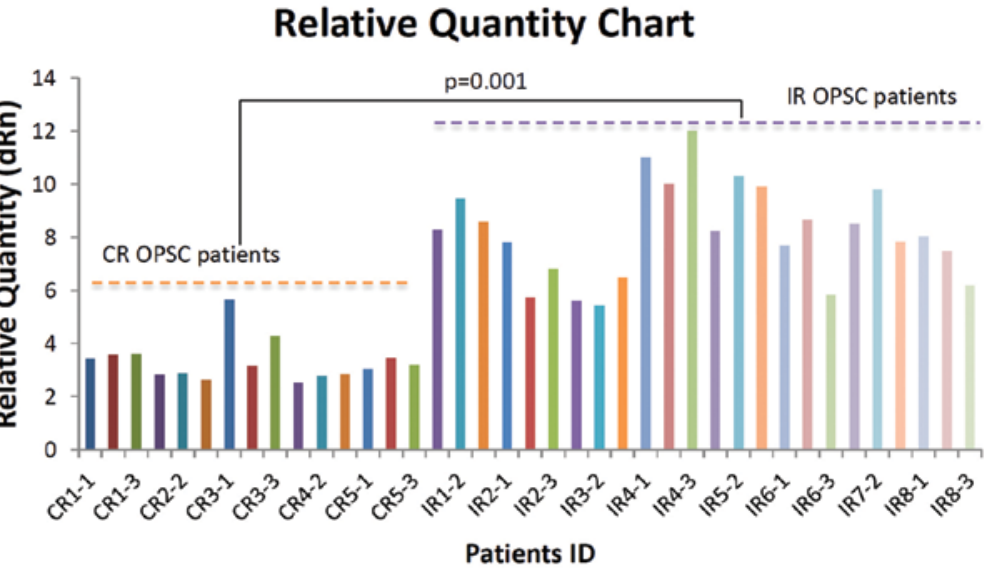

R3a

IR2a

C
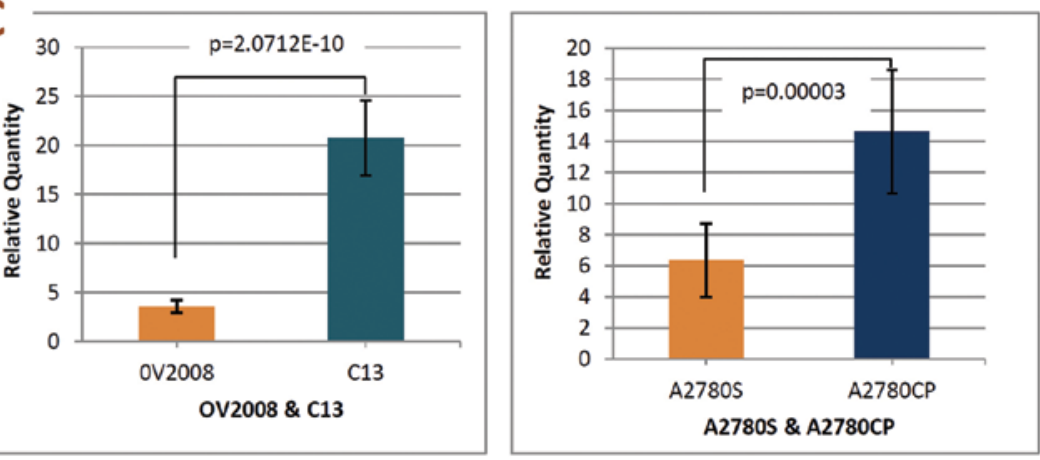

Figure 1. miR-224-5p expression is significantly increased in EOC patients and chemotherapy-resistant cell lines. (A) Hierarchical clustering of miRNA microarray analysis between complete response (CR) and incomplete response (IR) ovarian cancer patients. miR-224-5p is low-expressed with statistical significance ( $\mathrm{p}<0.0001)$ in the $\mathrm{CR}$ vs. IR group ( $\mathrm{n}=4$ for all groups, expression ratio of IR to $\mathrm{CR}=5.77062$, $\mathrm{p}=1.27 \mathrm{E}-05$, adjust $\mathrm{p}=2.97 \mathrm{E}-04)$. (B) Validation in set samples according to miR-224-5p expression by qRT-PCR. In keeping with microarray results, miR-224-5p was highly expressed in IR ( $\mathrm{n}=8$ ) with significance. $\mathrm{p}=0.001$, compared with the $\mathrm{CR}(\mathrm{n}=5)$. (C) In ovarian cancer cell lines, we also found that miR-224-5p expressions were all higher in cisplatin-resistant parental cell lines (C13 and A2780CP) compared to their cisplatin-sensitive variants (OV2008 and A2780S), and p-values were 2.0712E-10 and 0.000034, respectively $(\mathrm{n}=10$ for all groups). EOC, epithelial ovarian cancer.

all significant downregulation of miR-224-5p in OV2008 and A2780S cell lines compared with their cisplatin-resistant variants, respectively. These observations are consistent with the results in clinical ovarian cancer patient specimens.

Furthermore, we studied the effects of miR-224-5p expression patterns on chemosensitivity to cisplatin in vitro. After transfection with pre-miR-224-5p, the cell viabilities of OV2008 and A2780S appeared obviously higher than the mock or negative control group ( $\mathrm{p}<0.01$ for all, Fig. $2 \mathrm{~A}$ and B).

Effects of miR-224-5p on sensitivity of ovarian cancer cells to cisplatin-induced apoptosis. Cisplatin and other platinumbased cancer drugs destroy tumor cells by binding to DNA strands and forming cisplatin-DNA adducts. Vast DNA damage activates apoptosis when repair is impossible. Thus, we next examined whether miR-224-5p is involved in cisplatininduced chemoresistance by affecting apoptosis. To evaluate the effect of miR-224-5p expression on the chemosensitivity of ovarian cancer cells, we used cisplatin-sensitive parental cell line (OV2008 and A2780S) and its cisplatin-resistant variant $(\mathrm{C} 13$ and $\mathrm{A} 2780 \mathrm{CP})$ through transfection with premiR-224-5p (miRNA mimic), anti-miR-224-5p (inhibitor) or their scrambled negative controls, respectively. After transfection, cells were treated with cisplatin, followed by assessment of the activation of caspase- 3 and -7 and with TUNEL assays. As shown in Fig. 3A, cisplatin-induced apoptosis was reversed by miR-224-5p in tumor cells, as indicated by the decreased or increased caspase-3/7 activity observed in miR-224-5p upregulated or downregulated tumor cells, respectively (Fig. 3A).

Furthermore, TUNEL assays revealed that the ratio of apoptosis in cisplatin-resistant $\mathrm{C} 13$ cells after anti-miR-224-5p treatment was markedly increased compared to the negative control and mock group (Fig. 3B and C), and vice versa. These findings suggest that miR-224-5p may protect ovarian cancer cells from cisplatin-induced damage by preventing apoptosis.

miRNA target prediction and validations. To further understand the mechanism of miR-224-5p in regulating chemoresistance of ovarian cancer cells, we chose several computational approaches, including TargetScan, MicroCosm Targets and miRNA Target Visualization, to analyze its predicted targets. In order to retrieve the most relevant targets, we listed only the top significantly unique miRNAs which were predicted by these computational approaches, and we found PRKCD, 

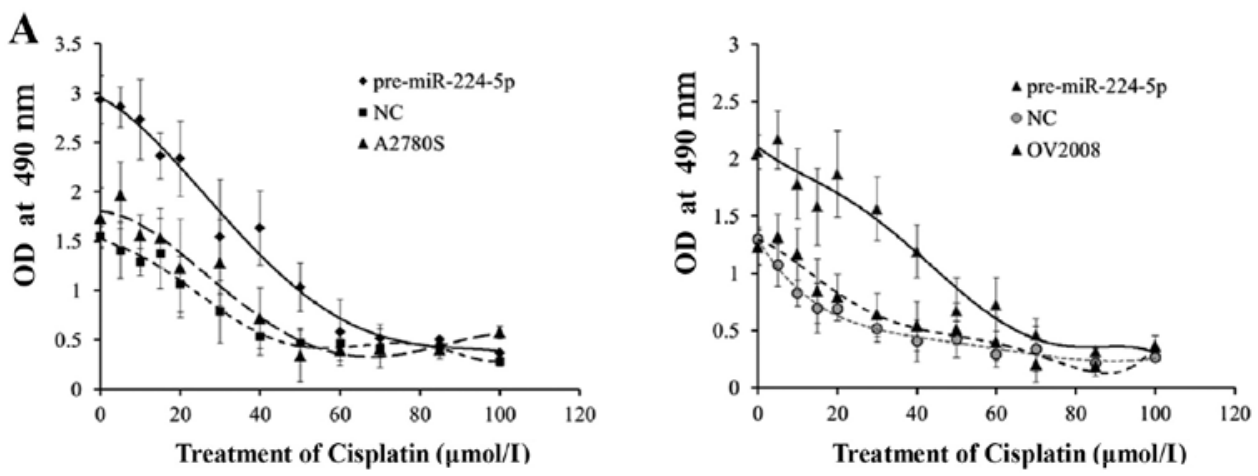

B
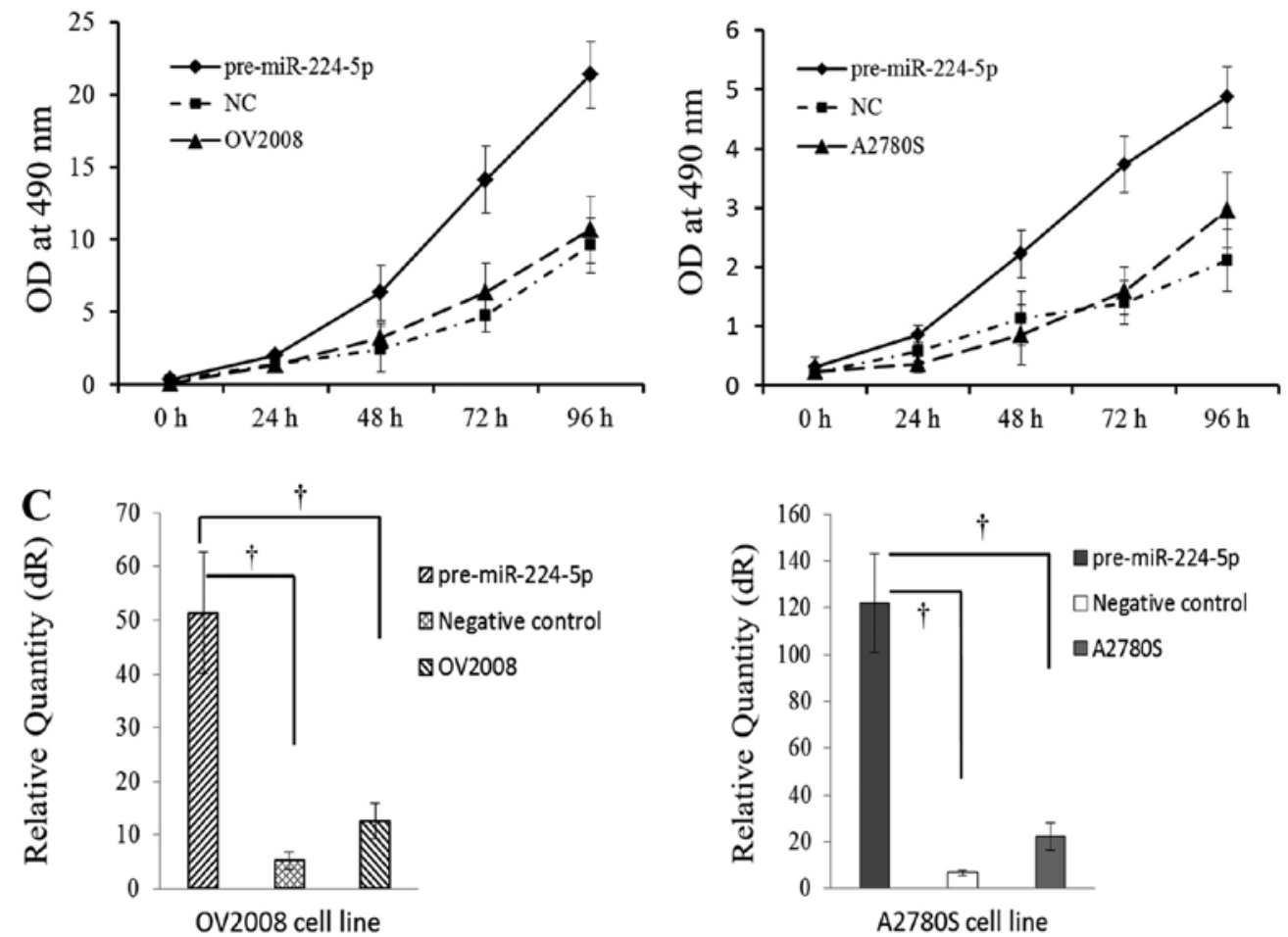

$\begin{array}{llll}\text { pre-miR-224-5p: } & + & - & - \\ \text { NC: } & - & + & -\end{array}$

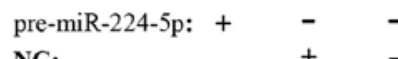

Figure 2. Upregulation of miR-224-5p increases ovarian cancer cell survival of chemosensitive cell line to cisplatin. (A) After transfection with pre-miR224-5p, negative control, and then treatment with gradient concentrations of cisplatin, the proliferation curves of both cisplatin-sensitive parental cell lines (OV2008 and A2780S) were all shifted to the right (n=10/each point). (B) After transfection with pre-miR-224-5p for 48 h, and then treatment with $20 \mu$ mol/1 cisplatin for $0,24,48$ and $96 \mathrm{~h}$, the cell viabilities of OV2008 and A2780S were all clearly increased, and their proliferation curves all shifted to the left compared with their negative control and mock cells ( $\mathrm{n}=10 /$ each point). (C) Real-time RT-PCR validated that pre-miR-224-5p transfections were well performed. ${ }^{\dagger}$, statistically significant difference of miR-224-5p, compared with either negative control or mock cells ( $\mathrm{p}<0.01$ for all).

whose gene product plays a role in both regulating chemoresistance and promoting apoptosis, is the common target of these approaches (Fig. 4). These results suggest that PRKCD may be a potential target gene of miR-224-5p in regulating cisplatin sensitivity.

We next validated this prediction with standard approaches such as PCR and western blot assay (Fig. 5B and C). qPCR analysis for miR-224-5p expression showed a significant downregulation in CR patients compared to IR cases $(\mathrm{p}=0.001$, Fig. 5B). However, its candidate target PRKCD negatively expressed between the same CR and IR patients. The correlation coefficients (r) and significant levels (p) were -0.309 and 0.035 , respectively.
To further examine whether PRKCD is the target of miR-224-5p at the in vitro level, cisplatin-resistant parental cell line (C13) and its sensitive variant (OV2008) were transfected with anti-miR-224-5p and pre-miR-224-5p, respectively. Western blot analyses revealed that miR-224-5p negatively regulated PRKCD expression at both cisplatin-resistant parental cell and its sensitive variant cell levels (Fig. 5C).

Prediction of survival rate in OPSC patients using miR-224-5p and PRKCD expression patterns. To elucidate the significance of miR-224-5p expression pattern in EOC chemosensitivity, we performed a retrospective study to investigate the relationship between miR-224-5p, PRKCD expression and chemosen- 

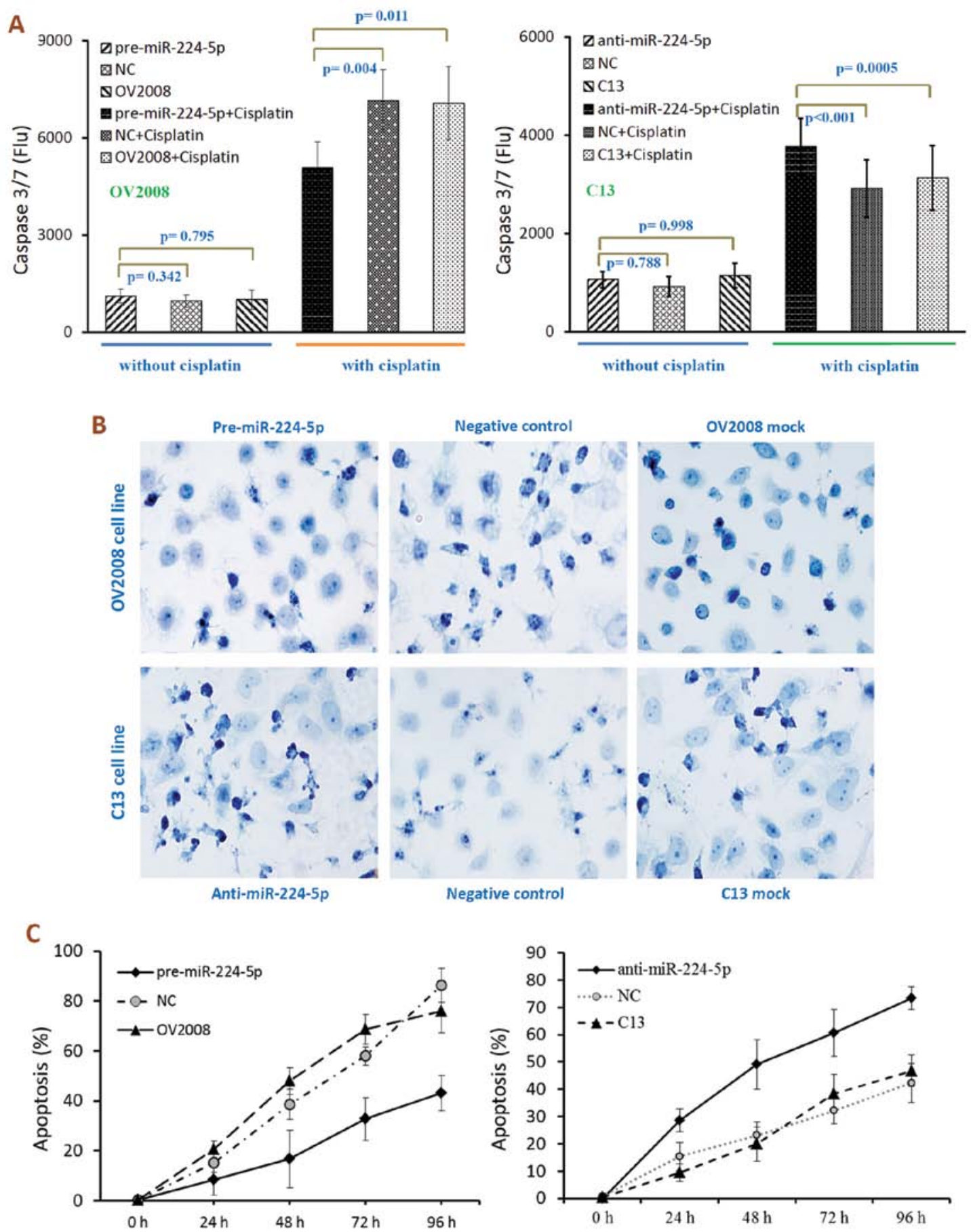

Figure 3. Effects of miR-224-5p on the sensitivity of ovarian cancer cells to cisplatin-induced apoptosis. (A) Overexpression or inhibition of expression of miR224-5p did not affect the activities of caspase-3/7 in OV2008 or C13, respectively. However, following treatment with cisplatin ( $20 \mu \mathrm{mol} / \mathrm{l}$ for $48 \mathrm{~h})$, the caspase Glo-3/7 apoptosis assay showed that the upregulation of miR-224-5p significantly suppressed cisplatin-induced apoptosis as indicated by the suppressed caspase-3/7 activity in OV2008 cell line ( $\mathrm{n}=10 /$ group), and vice versa. (B) TUNEL staining showed that treatment with cisplatin induced more apoptotic cells in anti-miR-224-5p transfection than negative control or mock group of C13 cell line. However, OV2008 became more resistant to cisplatin after being transfected with mimics of miR-224-5p. Original magnification, x400. (C) The percentage of apoptosis in pre-miR-224-5p-transfected OV2008 significantly decreased compared with its own NC-transfected and mock group ( $\mathrm{n}=10 /$ group), and vice versa.

sitivity in OPSC patients. A 41-sample training set (CR, 22; IR, 19) was used in analysis of leave-one-out cross predictions. Using a cut-off of 4.0225 and 8.1578 for miR-224-5p and PRKCD respectively, we found the highest Youden's index (Fig. 6A and B). Mann-Whitney U tests for statistical significance ( $<<0.001$ for all groups) demonstrated the capacity of the predictor to distinguish CR patients from IR patients. The mean area under ROC curve (AUC) values of miR-224-5p and PRKCD were 0.889 and 0.895 , respectively (Fig. 6C and D).

Based on the analysis of the above ROC research, KaplanMeier survival analysis indicated that high expression of miR-224-5p was significantly associated with poor overall survival of the OPSC patients as compared with the low miR-224-5p expression group, log-rank $\mathrm{p}=0.017$ (Fig. 6E). 


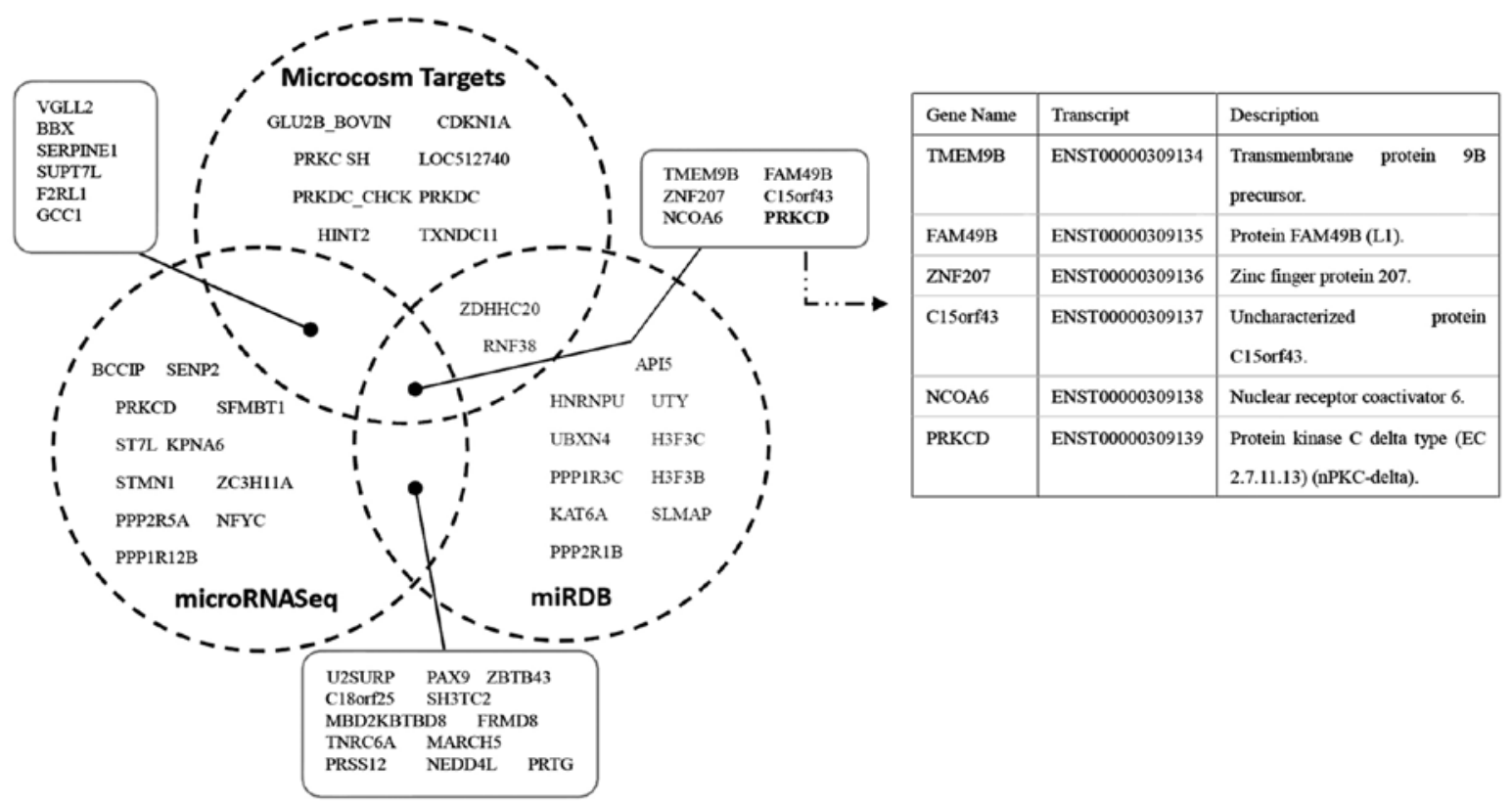

Figure 4. Integrating miRNA and mRNA data to identify genes associated with OPSC chemoresponsiveness. The Venn diagram represents the intersections of three computational database-predicted mRNA gene targets of miR-224-5p found to be associated with OPSC chemotherapy response in the present study. During the 6 co-target of these databases, PRKCD was found to play a role in both regulating chemoresistance and promoting apoptosis. OPSC, ovarian papillary serous carcinoma.

A miR-224-5p: 3'- $\boldsymbol{U} \boldsymbol{U} \mathrm{GCC} \boldsymbol{U} \boldsymbol{U} G \mathrm{G} G \boldsymbol{A} \boldsymbol{U} \subset \boldsymbol{A} \subset \boldsymbol{U} G \boldsymbol{A A} \subset$ - -5'

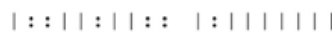

$|::||:||::|:|||||| \mid$

PRKCD, H. sapiens UTR 68-87: 5'- $\boldsymbol{A} \in \boldsymbol{T} G G G \boldsymbol{A} \subset \boldsymbol{T} G-\boldsymbol{T} G G \boldsymbol{T} \in \boldsymbol{A} \subset \boldsymbol{T} \boldsymbol{T} \subset-3$ '

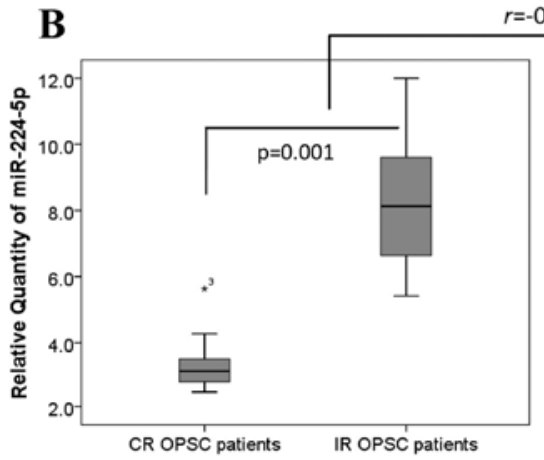

$=-0.309, p=0.035$

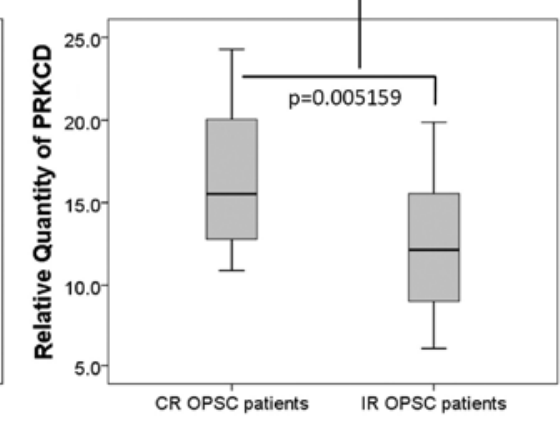

C
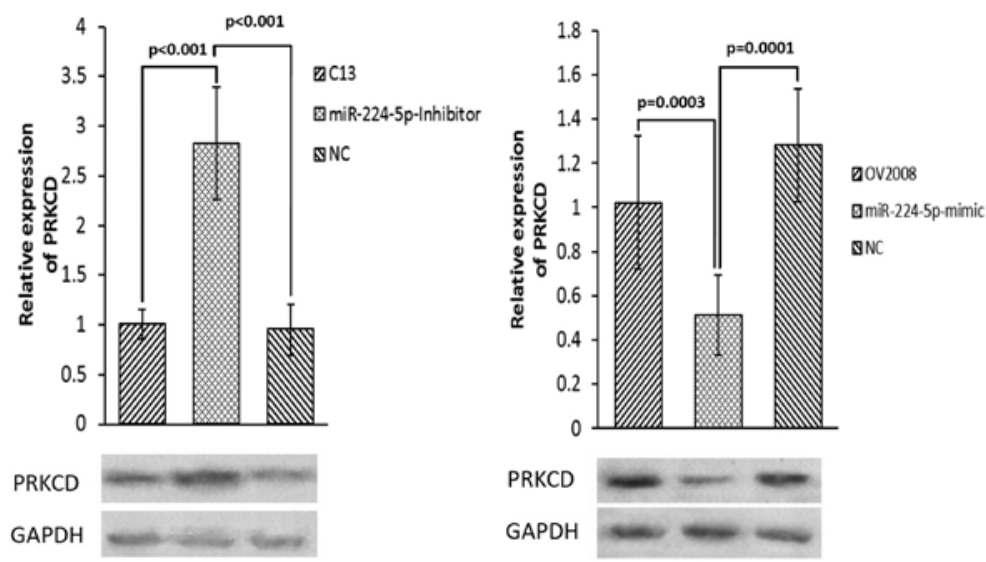

Figure 5. miR-224-5p negatively regulates PRKCD in vivo and in vitro. (A) Sequence alignment of human miR-224-5p with 3'-UTR of PRKCD. (B) Difference between miR-224-5p expression (left) and PRKCD mRNA level (right) in CR and IR patients, respectively. The correlation coefficients (r) and significant levels (p) were -0.309 and 0.035 between miR-224-5p and PRKCD expression in vivo. (C) Cisplatin-resistant parental cell line (C13) and its sensitive variant (OV2008) were transfected with anti-miR-224-5p and pre-miR-224-5p, respectively. Western blot analyses revealed that miR-224-5p negatively regulated PRKCD expressions at both cisplatin-resistant parental cell and its sensitive variant cell levels (C). 3'-UTR, 3'-untranslated region; CR, complete response; IR, incomplete response. 

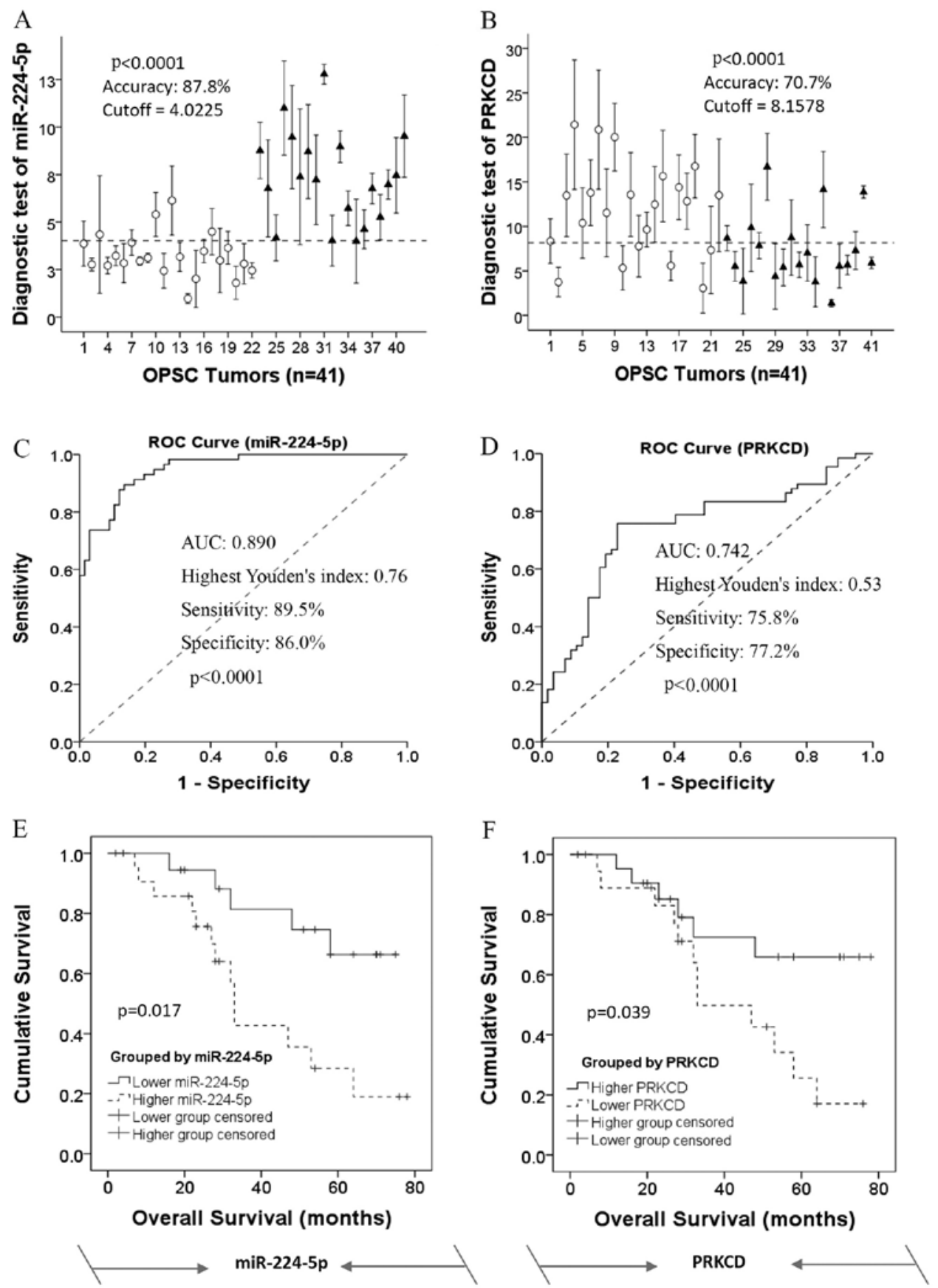

Figure 6. Kaplan-Meier curves showing the overall survival of OPSC patients stratified by expression levels of miR-224-5p and PRKCD. (A and B) Leaveone-out cross predictions of OPSC differentiation (white circle, CR patients; black triangle, IR patients) with (A) miR-224-5p and (B) PRKCD respectively, $\mathrm{n}=41$ for all; cut-offs were 4.0225 and 8.1578 with the highest Youden's index for each of them; accuracy, sensitivity and specificity were $87.8,89.5$ and $86.0 \%$ for miR-224-5p and 70.7, 75.8 and 77.2\% for PRKCD, respectively. (C and D) Receiver operating characteristic (ROC) curves of the predictions of OPSC differentiation according to (C) miR-224-5p and (D) PRKCD respectively, $\mathrm{n}=41$ for all. AUC values were 0.878 and 0.707 for miR-224-5p and PRKCD, respectively. (E and F) miR-224-5p and PRKCD serve as novel predictions and prognostic biomarkers for the predictive response to survival of OPSC patients. The overall survival result of a 41-sample training set divided into two sub-bits according to high and low expression level of (E) miR-224-5p and (F) PRKCD, as over or under the cut-off points, respectively. Diagnosis is based on the analysis of the above ROC research. Kaplan-Meier survival analysis indicated that the expression levels of miR-224-5p and with PRKCD may serve as prognostic biomarkers for OPSC patient response to overall disease-specific survival. CR, complete response; IR, incomplete response; OPSC, ovarian papillary serous carcinoma.

However, the result of PRKCD expression in Kaplan-Meier survival analysis was contrary to miR-224-5p (Fig. 6F).

These results indicate that the expression levels of miR-224-5p and with PRKCD attribute to revealing the chemoresistance of OPSC patients, and may serve as prognostic biomarkers for OPSC patient response to overall disease-specific survival.

\section{Discussion}

Platinum-based chemotherapy is the standard, first-line treatment for advanced-stage EOC. Almost all patients with EOC at the advanced stages receive the same treatment strategy, which is a platinum-based regimen, usually with cisplatin (15). However, the major impediment to a successful treat- 
ment is the drug resistance to chemotherapy. The purpose of the present study was to enhance our understanding of the biological mechanisms underlying the chemoresistance of human ovarian cancer and to identify possible therapies that can induce chemoresistance reversion. By comparing the miRNA microarray profiles of patients with CR and IR ovarian cancers, we found that miR-224-5p was significantly upregulated in IR patients. To examine the association of miR-224-5p with chemosensitivity of ovarian cancer cells in vitro, we used transient transfection of miR-224-5p (mimic or inhibitor) in ovarian cancer cell lines. We found that high level of miR-224-5p has potent promoting effects on ovarian cancer cell survival and inhibitory effects on apoptosis, and vice versa. Therefore, we identified miR-224-5p as a critical contributor to chemoresistance in epithelial ovarian cancer. Our conclusion is supported by both in vitro and in vivo experiments.

Recent evidence indicates that miRNAs may play important roles by affecting various pathways related to anticancer drug resistance, such as influencing the response to the conventional chemoagents, cisplatin or microtubule-targeting drugs (16-18). Therefore, identification of cancer-specific miRNAs as well as their targets is critical for understanding the roles of miRNAs in tumor genesis and may be important for defining novel chemotherapy (4).

To further understand the mechanism of miR-224-5p in regulating chemoresistance of ovarian cancer cells, we selected several computational approaches to analyze its predicted targets. In the present study, we focused on PRKCD, which plays a role in both regulating chemoresistance and promoting apoptosis. Previous studies revealed that the protein kinase $\mathrm{C}$ (PKC) signal pathway is a critical regulator of the chemosensitivity in cancers such as ovarian cancer, non-small cell lung cancer and prostate cancer (19-21).

PRKCD, known as protein kinase $C \delta$, is a PKC isozyme that acts as a substrate for caspase-3 (4). Its activity is believed to be required for apoptosis induced by DNA damaging agents such as cisplatin, mitomycin $\mathrm{C}$ and doxorubicin (22-24). Additionally, PRKCD has been reported to be a possible positive regulator of cisplatin-induced cell death in a gastric cancer cell line, leading to a modest increase in cisplatin uptake, and associated with the inhibition of cell cycle and tumor progression (25-28). Collectively, PRKCD has a negative effect on cell survival. In the present study, we demonstrated that miR-224-5p could negatively regulate the expression of PRKCD, and together with PRKCD, they can serve as novel predictors and prognostic biomarkers for OPSC patient response to overall disease-specific survival (Fig. 6). Therefore, the PRKCD pathway may be a molecular mechanism through which miR-224-5p exerts its functions as an oncogene and enhancer of chemoresistance to cisplatin in OPSC patients.

In conclusion, we report that alterations in miR-224-5p expression in ovarian cancer patients and cell lines confer differential chemosensitivity and that miR-224-5p may function as an enhancer of chemoresistance at least in part by targeting PRKCD. Therefore, the miR-224-5p-PRKCD interaction may become a biomarker for predicting chemosensitivity to cisplatin in patients with ovarian papillary serous carcinoma.

\section{References}

1. Zhao H, Ding Y, Tie B, et al: miRNA expression pattern associated with prognosis in elderly patients with advanced OPSC and OCC. Int J Oncol 43: 839-849, 2013.

2. Saldivar JS, Wu X, Follen M and Gershenson D: Nucleotide excision repair pathway review I: implications in ovarian cancer and platinum sensitivity. Gynecol Oncol 107 (Suppl 1): S56-S71, 2007.

3. Sorrentino A, Liu CG, Addario A, Peschle C, Scambia G and Ferlini C: Role of microRNAs in drug-resistant ovarian cancer cells. Gynecol Oncol 111: 478-486, 2008.

4. Chen Y, Ke G, Han D, Liang S, Yang G and Wu X: MicroRNA181a enhances the chemoresistance of human cervical squamous cell carcinoma to cisplatin by targeting PRKCD. Exp Cell Res 320: 12-20, 2014.

5. Zhang W, Dahlberg JE and Tam W: MicroRNAs in tumorigenesis: a primer. Am J Pathol 171: 728-738, 2007.

6. Kent OA and Mendell JT: A small piece in the cancer puzzle: microRNAs as tumor suppressors and oncogenes. Oncogene 25: 6188-6196, 2006.

7. Li Z, Hu S, Wang J, et al: MiR-27a modulates MDR1/Pglycoprotein expression by targeting HIPK 2 in human ovarian cancer cells. Gynecol Oncol 119: 125-130, 2010.

8. Duttagupta R, DiRienzo S, Jiang R, et al: Genome-wide maps of circulating miRNA biomarkers for ulcerative colitis. PLoS One 7: e31241, 2012

9. Metzeler KH, Maharry K, Radmacher MD, et al: TET2 mutations improve the new European LeukemiaNet risk classification of acute myeloid leukemia: a cancer and leukemia group B study. J Clin Oncol 29: 1373-1381, 2011.

10. Enerly E, Steinfeld I, Kleivi K, et al: miRNA-mRNA integrated analysis reveals roles for miRNAs in primary breast tumors. PLoS One 6: e16915, 2011.

11. Powers MP, Alvarez K, Kim HJ and Monzon FA: Molecular classification of adult renal epithelial neoplasms using microRNA expression and virtual karyotyping. Diagn Mol Pathol 20: 63-70, 2011.

12. Bansal N, Marchion DC, Bicaku E, et al: BCL2 antagonist of cell death kinases, phosphatases, and ovarian cancer sensitivity to cisplatin. J Gynecol Oncol 23: 35-42, 2012.

13. Miller AB, Hoogstraten B, Staquet M and Winkler A: Reporting results of cancer treatment. Cancer 47: 207-214, 1981.

14. Rustin GJ, Nelstrop AE, Bentzen SM, Piccart MJ and Bertelsen K: Use of tumour markers in monitoring the course of ovarian cancer. Ann Oncol 1: 21-27, 1999.

15. Dressman HK, Berchuck A, Chan G, et al: An integrated genomic-based approach to individualized treatment of patients with advanced-stage ovarian cancer. J Clin Oncol 25: 517-525, 2007.

16. Giovannetti E, Erozenci A, Smit J, Danesi R and Peters GJ: Molecular mechanisms underlying the role of microRNAs (miRNAs) in anticancer drug resistance and implications for clinical practice. Crit Rev Oncol Hematol 81: 103-122, 2012.

17. Tian W, Chen J, He H and Deng Y: MicroRNAs and drug resistance of breast cancer: basic evidence and clinical applications. Clin Transl Oncol 15: 335-342, 2013.

18. Kanakkanthara A and Miller JH: MicroRNAs: novel mediators of resistance to microtubule-targeting agents. Cancer Treat Rev 39: 161-170, 2013.

19. Qamar L, Davis R, Anwar A and Behbakht K: Protein kinase C inhibitor Gö6976 augments caffeine-induced reversal of chemoresistance to cis-diamminedichloroplatinum-II (CDDP) in a human ovarian cancer model. Gynecol Oncol 110: 425-431, 2008.

20. Clark AS, West KA, Blumberg PM and Dennis PA: Altered protein kinase $\mathrm{C}$ (PKC) isoforms in non-small cell lung cancer cells: PKCס promotes cellular survival and chemotherapeutic resistance. Cancer Res 63: 780-786, 2003.

21. Sumitomo M, Asano T, Asakuma J, Asano T, Nanus DM and Hayakawa M: Chemosensitization of androgen-independent prostate cancer with neutral endopeptidase. Clin Cancer Res 10: 260-266, 2004

22. Basu A and Akkaraju GR: Regulation of caspase activation and cis-diamminedichloroplatinum(II)-induced cell death by protein kinase C. Biochemistry 38: 4245-4251, 1999.

23. Blass M, Kronfeld I, Kazimirsky G, Blumberg PM and Brodie C: Tyrosine phosphorylation of protein kinase $\mathrm{C} \delta$ is essential for its apoptotic effect in response to etoposide. Mol Cell Biol 22: 182-195, 2002 
24. Basu A, Woolard MD and Johnson CL: Involvement of protein kinase $C-\delta$ in DNA damage-induced apoptosis. Cell Death Differ 8: 899-908, 2001.

25. Iioka Y, Mishima K, Azuma N, et al: Overexpression of protein kinase $\mathrm{C} \delta$ enhances cisplatin-induced cytotoxicity correlated with p53 in gastric cancer cell line. Pathobiology 72: 152-159, 2005.

26. Basu A and Evans RW: Comparison of effects of growth factors and protein kinase $\mathrm{C}$ activators on cellular sensitivity to cisdiamminedichloroplatinum(II). Int J Cancer 58: 587-591, 1994.
27. Gentilin E, Tagliati F, Filieri C, et al: miR-26a plays an important role in cell cycle regulation in ACTH-secreting pituitary adenomas by modulating protein kinase $\mathrm{C} \delta$. Endocrinology 154: 1690-1700, 2013.

28. Hernández-Maqueda JG, Luna-Ulloa LB, Santoyo-Ramos P, Castañeda-Patlán MC and Robles-Flores M: Protein kinase C delta negatively modulates canonical Wnt pathway and cell proliferation in colon tumor cell lines. PLoS One 8: e58540, 2013. 